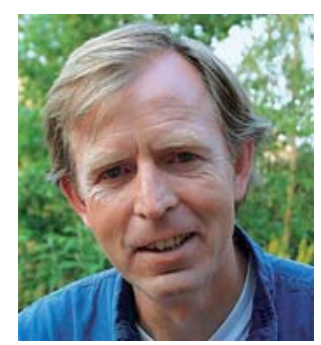

\title{
Ring the Bells!
}

In the previous editorial we looked at the autism "epidemic" and its possible causes, and several authors in issue 1/2011 and in this subsequent issue share successful homeopathic solutions and treatment protocols. The causes seem real, as does the problem, as do the solutions. It is wonderful that homeopathy can play such a meaningful role in the life of children and parents here.

And then I read how a twelve-year-old finished high school by the age of eight and currently is questioning Einstein's relativity theory. According to his professors he is asking the right questions.

I also see how a young man is being flown over Rome in a helicopter. Once back and seated behind a large piece of paper he is able to make a drawing of the city in amazing detail.

Another young child reproduces on the piano a complicated piece of music after only listening to it once, where this takes others hours and hours of blood, sweat and tears to master it.

There are many more examples of children with amazing skills, that we have labelled autistic or whatever we use to state they are different from us, the so-called healthy norm. Could it be that they don't need social skills?

Autism is not the only "handicap" that seems to have another side to it.

I see a young man moving an audience to tears while playing the piano with his toes. Who says you need arms?

I see another young man without any limbs being a motivational speaker for an audience full of four-limbed people. Perhaps he's the only healthy person in that hall. Who says we need limbs?

Another person had muscular dystrophy and could only move some muscles in his face. Besides that he was deaf and blind. In 1992 he was told he would die in a matter of months at the most. He lived another 18 years, studied law and got a girlfriend. You could talk to him by using his cheek as if it were a keyboard. He "spoke" at medical congresses about the quality of life. He showed how valuable life still can be, even if we no longer recognise it and feel that death is mercy.

I see severely handicapped people having smiles that suggest they are in a constant state of grace. They open the hearts of many and some call them star people.

Summing this up fills me with gratitude for the body I have and for the gift these beautiful people give us by opening our hearts and minds. If they can have such a meaningful life and give so much to the world, how about us? Let's Ring the Bells!

Ring the bells that still can ring

Forget your perfect offering

There is a crack in everything

That's how the light gets in.

Leonard Cohen, “Anthem"

Harry van der Zee

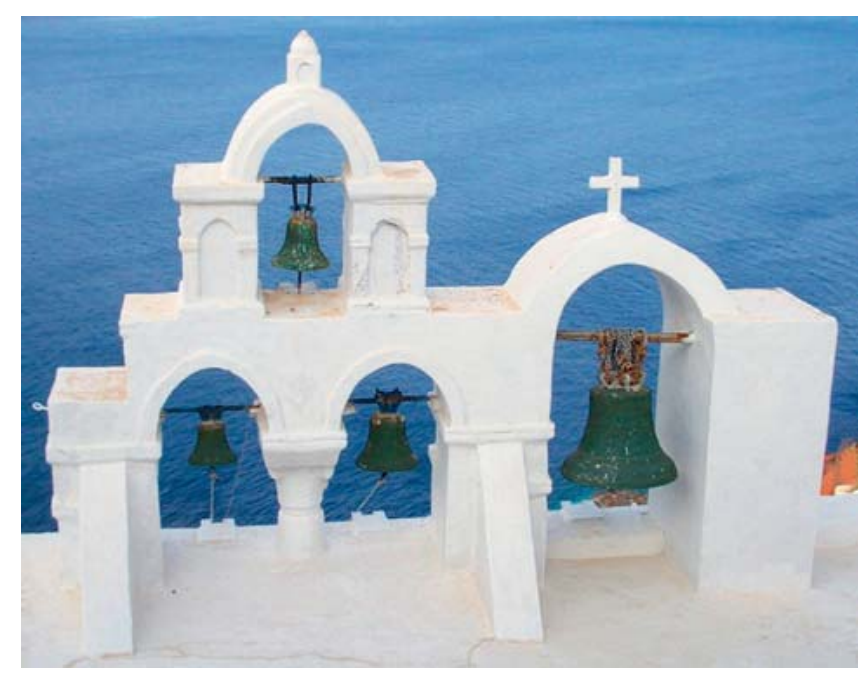

Church bells at Santorini. 